\title{
Veterinary Drug Residues in Food-animal Products: Its Risk Factors and Potential Effects on Public Health
}

\section{Takele Beyene*}

"Department of Biomedical Sciences, College of Veterinary Medicine and Agriculture, Addis Ababa University, P.O.Box 34, Bishoftu, Ethiopia

*Corresponding author: Takele Beyene, Department of Biomedical Sciences, College of Veterinary Medicine and Agriculture, Addis Ababa University, P.O.Box 34, Bishoftu, Ethiopia, +251-912-82-82-53; Fax: +251 1143399 33; E-mail: takele.beyene@aau.edu.et

Rec date: Aug 14, 2015; Acc date: Dec 08, 2015; Pub date: Dec 11, 2015

Copyright: $\odot 2015$ Beyene T, et al. This is an open-access article distributed under the terms of the Creative Commons Attribution License, which permits unrestricted use, distribution, and reproduction in any medium, provided the original author and source are credited.

\begin{abstract}
The use of veterinary drugs in food-producing animals has the potential to generate residues in animal derived products (meat, milk, eggs and honey) and poses a health hazard to the consumer. There are many factors influencing the occurrence of residues in animal products such as drug's properties and their pharmacokinetic characteristics, physicochemical or biological processes of animals and their products. The most likely reason for drug residues might be due to improper drug usage and failure to keep the withdrawal period. The major public health significances of drug residue are development of antimicrobial drug resistance, hypersensitivity reaction, carcinogenicity, mutagenicity, teratogenicity, and disruption of intestinal normal flora. The residual amount ingested is in small amounts and not necessarily toxic. However, there is limited information on the magnitude of veterinary drug residue worldwide. Hence, an extensive work has to be carried out to determine the magnitude of the problem, to prevent the occurrence of veterinary drug residues, and to familiarize all animal health professionals with the knowledge of pharmacokinetics, pharmacodynamics and toxicological effects of veterinary drugs to minimize the potential public health hazards due to drug residues in food of animal origin.
\end{abstract}

Keywords: Food-animal products; Public health significance; Residues; Toxicological effects and veterinary drugs

\begin{abstract}
Abbreviations
ADI: Acceptable Daily Intake; CVM: Centre for Veterinary Medicine; DNA: Deoxyribonucleic acid; ELU: Extra Label-Drug Use; EU: European Union; FDA: Food and Drug Administration; JECFA: Joint FAO/WHO Expert Committee on Food Additives; MRL: Maximum Residual Limit; NOEL: No Observed Effect Level; Vd: Volume of Distribution; VMPs: Veterinary Medicine Products; WHO: World Health Organization
\end{abstract}

\section{Background}

Human health is related directly to the environment [1], and in particular the nature and quality of the food [2]. Quality of food from animal products is widely concerning public health agencies around the world since veterinary drugs have played an important role in the field of animal husbandry and agro-industry, and increasing occurrence of residues, and resistance have become interesting issues [3]. Veterinary drugs or veterinary medicinal products (VMPs) are critically needed to meet the challenges of providing adequate amounts of food for the growing world population [4] as drugs improve the rate of weight gain, improve feed efficiency, or prevent and treat diseases in food producing animals [4,5]. However, the benefit of improved productivity from the use of VMPs in food producing animals is not obtained without the risk associated with VMPs residues that remain in the tissues of treated animals at the time of slaughter $[4,6]$ or residues in animal derived products and poses a health hazard to the customer. Antibacterial drugs and hormonal growth promoters are the main VMPs that potentially contaminate foods of animal origin [7].
Hence, veterinary drug or VMPs residue is one of many global issues concerning food contamination $[2,3]$.

Residues, as defined by the European Union (EU) and the Center for Veterinary Medicine, an agency under the Food and Drug Administration (FDA/CVM) in the USA are "pharmacologically active substances (whether active principles, recipients or degradation products) and their metabolites which remain in foodstuffs obtained from animals to which the VMPs in question has been administered" [8]. Under the normal physiological conditions, following administration of a drug to an animal, most drugs are metabolized in order to facilitate elimination, and to a large extent detoxification as well. In general, most of the parent product and its metabolites are excreted in urine and a lesser extent via faeces [9]. However, these substances may also be found in milk and eggs, and in the meat [10].

Rationally, there is no product coming from a treated animal should be consumed unless the entire drug administered has been eliminated. This is called zero tolerance, where this concept is in fact equivalent to the idea of total absence of residual amounts. However, because of the improvement of analytical techniques, which meant that the value of zero became smaller and smaller that depicts the limits corresponding to the sensitivities of parts per million (ppm), parts per billion (ppb) and parts per trillion (ppt). As a result, by using the high efficacy analytical methods, for instance, using high performance liquid chromatography, it can be concluded that there are nearly always detectable residues, but such residues are at an extremely low concentration and they are not inevitably toxic [11]. This review provides an overview of the risk factors for development of drug residues, their global incidence rates and public health significances; and to present a safety evaluation and avoidance measures of drug residues in food of animal origin. 


\section{Risk Factors for the Development of Residue in Food- producing Animal}

Veterinary drug residues are one of the major problems for food contamination [12]. VMPs and agricultural chemicals used according to label directions should not result in residues at slaughter. However, possible reasons for such residues include: Not following recommended label directions or dosage (extra-label usage); not adhering to recommended withdrawal times; administering too large a volume at a single injection site; use of drug-contaminated equipment, or failure to properly clean equipment used to mix or administer drugs; dosing, measuring, or mixing errors; allowing animals access to spilled chemicals or medicated feeds; animal effects- age, pregnancy, congenital, illness, allergies; chemical interactions between drugs; variations in water temperature for fish species; environmental contamination; and improper use of agricultural chemicals such as pesticides [13].

Veterinary drugs or VMPs residues usually accumulate in the liver or kidney rather than other tissues. It has been noted that different residue levels can be found in different tissue positions such as site and route of administration [12]. The most likely reason for drug residues may result from human management, such as improper usage, including extra-label or illegal drug applications. However, the most obvious reason for unacceptable residues might be due to failure to keep to the withdrawal period including using overdose and long acting drugs [14]. Inadequate good sanitary care during animal or product transportation, including the cross contamination of animal feeding stuffs with inadvertently applied drugs, environmental and animal to animal transfer of drugs may also cause residues. Risk factors responsible for the development of residue are:

\section{Age of animal}

Weaning status and, to a lesser extent, the age of the animal affect drug disposition [15]. For instance, the study conducted on comparisons of the pharmacodynamics of norfloxacin nicotinate between weaning and unweaned calves revealed that the distribution of the drug did not differ between the two groups of calves, but the total body clearance time was increased in weaned calves, possibly due to increased weight from the presence of rumen fluid [16]. Calves fed grain had shorter clearance times (approximately four days) for sulfamethazine than unweaned calves. The elimination half-life of tindazole is shorter in unweaned calves than in adult cows, while the elimination half-life of apramycin is longer in calves than in adult cattle, possibly due to the immaturity of the drug clearance system [17].

\section{Feeding}

Diet can affect the bioavailability of drugs [18]. For instances, study conducted to determine the effects of diet content on the bioavailability of orally administered fenbendazole to cattle and Indian buffalo and fed dry hay either with or without fresh green herbage showed that animals receiving feed containing fresh herbage had lowered bioavailability of the drug. Fenbendazole stays in the rumen and is progressively released with digesta, and the presence of fresh herbage increases gut activity and the flow rate of digesta, which depletes the available stores of fenbendazole in the rumen. In regard to feeds, actual gut contents can also affect drug uptake and pharmacodynamics $[17,19,20]$.

\section{Disease status}

The disease status of an animal can affect the pharmacokinetics of drugs administered, which can influence the potential for residues [9]. This can occur either when the disease affects the metabolic system (and consequently drug metabolism), or when the presence of infection and/or inflammation causes the drug to accumulate in affected tissues. For example, cattle with acutely inflamed mastitis quarters, apramycin penetrates these areas of the body, and concentrations of the drug have been observed at ten times over the level recorded from cows without mastitis. Ketoprofen levels in milk increase during clinical mastitis where there is an influx of serum components into the udder. In calves with experimentally induced fasciolosis, the elimination half-life of antipyrine was slightly increased, but was slightly decreased for erythromycin and statistically significant decrease for oxytetracycline. The proposed mechanisms for these changes were the changes in liver function by fasciolosis, which changed the processing of drugs through the liver [21].

\section{Pharmacokinetics}

The term pharmacokinetics refers to the movement of drug into, through and out of the body: the time course of its absorption, bioavailability, distribution, metabolism, and excretion [9].

\section{Absorption}

It is described as the process, which a compound passes from its site of administration into the bloodstream [9]. Absorption is influenced by many factors such as the properties of cell membrane, drug properties and route of administration and physiopathological state of the animal. An indication of the rate of drug absorption is obtained from the peak plasma concentration (Cmax) and time reaching the maximum concentration $(\operatorname{Tmax})[9,22]$.

\section{Distribution}

It is the process whereby a drug is transported to all the tissues and organs. After entering the systemic circulation, in whatever route of administration, drugs are conveyed throughout the body and reach their site of action. There are four major factors responsible for the extent and rate of distribution. These are the physicochemical properties of the drug, the concentration gradient established between the blood and tissue, the ratio of blood flow to tissue mass, and the affinity of the drug for tissue constituents and serum protein binding. Only the fraction free form (unbound) of the drug is capable of exiting the circulation to distribute through the body and exert activity at the site of action. The parameter, which defines the process of distribution, is the volume of distribution [23].

\section{Metabolism (Biotransformation)}

It is the principal mechanism of elimination for the transformation of drugs or xenobiotics into metabolites of the chemical reaction. Hepatocytes play an extremely important role in the metabolism of drugs and xenobiotic-compounds that are foreign to the body, some of which are toxic. The kidneys are responsible ultimately to dispose of these substances, but for effective elimination, the drug or its metabolites must be made hydrophilic (polar, water-soluble). This is because reabsorption of a substance by the renal tubules is dependent on its hydrophobicity. The more hydrophobic (non-polar, lipid-soluble) substance is, the more likely it will be reabsorbed. Many drugs and 
Page 3 of 7

metabolites are hydrophobic, and the liver converts them into hydrophilic compounds by using the two classes of enzymatic pathways of biotransformation; phase I (non-synthesis) and phase II (conjugation). Phase I corresponds to functionalization processes including oxidation, reduction, hydrolysis, hydration and isomerization reactions. Phase II reactions involve conjugation of the drug or phase I metabolite with the endogenous substrate such as glucuronic acid, sulfate, acetate and methyl group. Although some drugs are eliminated from the body by uncharged, most drugs undergo metabolism where the liver is the main organ of reaction. In addition, the liver's function may change the drug's form to be inactive and easy to excrete but some drugs may be converted to an activating form [22].

\section{Excretion}

It is the process by which the parent drug or its metabolites are removed from the body fluids. The kidney is the most important site of drug excretion. There are three renal mechanisms; glomerular filtration, carrier mediated proximal tubular secretion and $\mathrm{pH}$ dependent, passive tubular resorption in the distal nephron. Renal insufficiency usually significantly affects drug excretion. The systemic clearance and elimination half-life are important parameters referring to the overall rate of elimination (metabolism and excretion). Although most compounds are excreted primarily by the renal, some drugs are partially or completely excreted through the bile. It has been reported that there is an extensive species variation among animals in their general ability to excrete drugs in the bile; example, chicken are characterized as good biliary excretes, whereas sheep and rabbit are characterized as moderate and poor excretes [22].

\section{Extra-label drug use (ELU)}

Extra-label Drug Use (ELU) refers to the use of an approved drug in a manner that is not in accordance with the approved label directions. ELU occurs when a drug only approved for human use is used in animals, when a drug approved for one species of animal is used in another, when a drug is used to treat a condition for which it was not approved, or the use of drugs at levels in excess of recommended dosages $[9,24]$. For instances, the use of phenobarbital (a drug only approved for use in humans) to treat epilepsy in dogs and cats; the use of ivermectin in dogs and cats (an antiparasitic only approved for use in cattle); and the use of enrofloxacin solution as a topical ear medication (only approved for use as an injection) are the common ELU in veterinary medicine [25].

There are conditions for ELU in food animals. For example, when considering ELU of an approved human drug in food animals: the veterinarian must have medical rational for the use; the veterinarian may not use an approved human drug if an animal drug approved for use in food-producing animals can be used instead for the particular ELU; and if scientific information on the human food safety aspect of the use of the drug in food-producing animals is not available, the veterinarian must take appropriate measures to assure that the animal and its food products will not enter the human food supply [25].

The families of drugs (both in animals and humans), and substances currently prohibited for ELU in all food producing animals, (including horses intended for human food) are chloramphenicol, clenbuterol, diethylstilbestrol (DES), dimetridazole, ipronidazole, furazolidone, nitrofurazone, sulfonamide drugs in lactating dairy cattle (except approved use of sulfadimethoxine, sulfabromo-methazine, and sulfamethoxypyridazine, fluoroquinolones, and glycopeptides) [26].

\section{Improper withdrawal time}

The withdrawal time (also known as the depletion or clearance period) is the time for the residue of toxicological concern to reach a safe concentration as defined by the tolerance. Depending on the drug product, dosage form, and route of administration, the withdrawal time may vary from a few hours to several days or weeks. It is the interval necessary between the last administration to the animals of the drug under normal condition of used and the time when treated animal can be slaughtered for the production of safe foodstuffs [17].

\section{Incidence of veterinary drug residues}

In many countries, VMPs may be used indiscriminately for the treatment of animal diseases or they may be used as feed additives for domestic animals [27]. Different studies have been conducted by Zuo et al. [28] in Pennsylvania) and Babapour et al. [29] in Iran to show the incidence rate of VMPs residue in different parts of the world. The studies revealed that low level of heavy metals and gentian violet residue from catfish was detected $[28,30]$. Other studies conducted in Nigeria also revealed the detection of antimicrobial drug residues in commercial eggs [31], in meat from slaughtered cattle [32]. Furthermore, oxytetracycline and penicillin G from milk [33], and tetracycline from cattle beef [34] were also detected in Ethiopia.

The ongoing threat of antibiotic contamination is one of the biggest challenges to public health that is faced by the human population worldwide. Such residues are spreading rapidly, irrespective of geographical, economical, or legal differences between countries [35]. Additionally, the study reported in 2004 by EU also revealed that the majority of residues confirmed in animals were antibacterial agents [8].

Currently, the joint FAO/WHO Expert Committee on Food Additives (JECFA) has also reported various veterinary drugs and other environmental substances residues in a series of working documents. Additionally, the JECFA has been participating in further evaluating the safety of residues of veterinary drugs in food and in establishing acceptable daily intakes (ADIs), and recommend maximum residue limits (MRLs) for substances when they are administered to food-producing animals in accordance with good veterinary practice in the use of veterinary drug [36].

\section{Potential effect of veterinary drug residues on public health}

Drug low-level contamination generally may not generate a violation problem on public health. However, extensive use of drugs may increase the risk of an adverse effect of residues on the customer including the occurrence of antibiotic resistance [14,37] and hypersensitivity reaction [37]. Therefore, prudent use of drugs in the manner of preventing feed contamination is necessary [38].

\section{Development of drug resistance}

Human health can either affect through residues of drugs in food of animal origin, which may cause direct side effects [14], or indirectly, through selection of antibiotic resistance determinants that may spread human pathogen $[37,39,40]$. Resistant microorganism can get access to human, either through direct contact [39] or indirectly via milk, meat, and or egg. As the bacteria of animal origin, they may either colonize human endogenous flora or superimpose and additional load to the reservoir of resistance genes already present in man. The potential for 
animal to human transfer of resistance is existed. Clearly, the use of antibiotic in livestock production has been associated with the development of human antibiotic resistance [39,40]. The animal fed with the low prophylactic level of antibiotic may develop bacteria evolving resistance to this antibiotic during the preparation or consumption of food of animal origin [41]. It has been documented that human develop drug resistant bacteria such as Salmonella, Campylobacter, and Staphylococcus from food of animal origin [39]. Examples of drugs that have been shown to cause the growth of resistant bacteria in food of animal are fluoroquinolones and avaoparin. The resistance of microorganisms, arising from subtherapeutic uses of penicillin, tetracyclines, and sulfa drugs; in agriculture is suggested by the WHO to be a high priority issue [41].

\section{Drug hypersensitivity reaction}

Drug hypersensitivity is defined as an immune mediated response to a drug agent in a sensitized patient, and drug allergy is restricted to a reaction mediated by IgE. An allergic or hypersensitive effect following administration of a drug (i.e., drug allergy is quite similar to that typified by allergic response to protein, carbohydrate, and lipid macromolecules. Allergic reactions to drugs may include anaphylaxis, serum sickness, cutaneous reaction, a delayed hypersensitivity response to drugs appear to be more commonly associated with the antibiotics, especially of penicillin ([42]. About $10 \%$ of the human population is considered hypersensitive to an amount of a substance, including penicillin, but in animals, the extent of hypersensitive to, the drug is not well known [9]. Certain macrolides may also in exceptional be responsible for liver injuries, caused by a specific allergic response to macrolide modified hepatic cells [35].

\section{Carcinogenic effect}

The term carcinogen refers to an effect produced by a substance having carcinogenic activity [43] considerable confusion has existed because a carcinogen applies to substances that are so varied in their qualitative and quantitative characteristics. The potential hazard of carcinogenic residues is related to their interaction or covalently binding to various intracellular components such as proteins, deoxyribonucleic acid (DNA), ribonucleic acid (RNA), glycogen, phospholipids, and glutathione [44].

\section{Mutagenic effect}

The term mutagen is used to describe chemical or physical agents that can cause a mutation in a DNA molecule or damage the genetic component of a cell or organisms. Several chemicals, including alkalizing agents and analogous of DNA bases, have been shown to elicit mutagenic activity [45]. There has been increasing concern that drugs as well as environmental chemicals may pose a potential hazard to the human population by production of gene mutagen or chromosome breakage [46] that may have adversely affects human fertility [47].

\section{Teratogenic effect}

The term teratogen applies to drug or chemical agent that produces a toxic effect on the embryo or fetus during a critical phase of gestation. Consequently, a congenital malformation that affects the structural and functional integrity of the organism is produced. The well-known thalidomide incident involving a number of children in
Europe was a direct testimony to the hazard that may occur when such agent is administered during pregnancy [46]. Of the anthelmintics, benzimidazole is embryo toxic and teratogenic when given during early stage of pregnancy because of the anthelminthic activity of the drug $[44,48]$. In addition to embryo toxicity including teratogenicity, the benzimidazole drug of oxfendazole, has also exhibited a mutagenic effect [48].

\section{Disruption of Normal Intestinal Flora}

The bacteria that usually live in the intestine acts as a barrier to prevent incoming pathogen from being established and causing diseases. Antibiotics may reduce the total number of the bacteria or selectively kill some important species. The broad-spectrum antimicrobials may adversely affect a wide range of intestinal flora and consequently cause gastrointestinal disturbance [49]. For example, use of drugs like, flunixin, streptomycin [50], and tylosin in animals, and also use of vancomycin, nitroimidazole, and metronidazole in humans [49] are known for this effect

\section{Safety Evaluation for VMPs Residue}

\section{Acceptable daily intake (ADI)}

It is the amount of a substance that can be ingested daily over a lifetime without appreciable health risk. Calculation of ADI is based on an array of toxicological safety evaluation that takes into acute and long-term exposure to the drug and its potential impact [51]. If the drug is not a carcinogen, the no observed effect level (NOEL) of the most sensitive effect in the most sensitive species divided by a safety factor is used to determine an ADI for drug residues. The FDA will calculate the safe concentration for each edible tissue using the ADI, the weight in $\mathrm{kg}$ of an average adult $(60 \mathrm{~kg})$, and the amount of the product eaten per day in grams as follows [26].

Safe concentration=[ADI $(\mu \mathrm{g} / \mathrm{kg} /$ day $) \times 60 \mathrm{~kg}] /[$ Grams consumed $/$ day].

\section{Maximum residue limit (MRL)}

It is defined as the maximum concentration of a residue, resulting from the registered use of an agricultural or veterinary chemical, which is recommended to be legally permitted or recognized as acceptable in or on a food, agricultural commodity, or animal feed. The concentration is expressed in milligrams per kilogram of the commodity (or milligrams per liter the case of a liquid commodity) [52].

\section{Calculating withdrawal time}

The withdrawal period or the milk discards time is the interval between the time of the last administration of a veterinary drug and the time when the animal can be safely slaughtered for food or the milk can be safely consumed. The withdrawal period is determined when the tolerance limit on the residue concentration is at or below the permissible concentration. A tolerance limit provides an interval within which a given percentile of the population lies, with a given confidence that the interval does contain that percentile of the population [53].

Withdrawal times are determined in edible, target tissues by FDA/CVM during the drug approval process. These target tissues are 
most commonly the liver or kidney. As the primary organs of elimination, they will typically display a residue for the longest time. During withdrawal studies, the target organ is determined and animals are sampled at various times after drug administration is stopped. Statistical procedures are used to determine when almost every animal given the drug would be below the drug tolerance concentration in the target organ. A muscle tolerance has also been established for some drugs. For those drugs for which only a kidney or liver tolerances has been established, if a violative residue is found in the target organ, the whole carcass would need to be discarded. On the other hand, for the drugs for which a muscle tolerance has been established, even if a violative residue is found in the kidney or liver a violative residue is not found in the muscle, the carcass would not need to be discarded. The disposition of such carcasses cannot be determined by testing of liver, kidney, and muscle is completed [54].

\section{Residue Avoidance}

\section{Pharmacological principles}

To implement an effective residue avoidance program in a food animal practice, a veterinarian must be aware of pharmacological principles of many drugs. Half-life ( $\mathrm{t} 1 / 2)$ is the time it takes to remove $50 \%$ of the drug from the animal and used to estimate withdrawal time. A drug with a large volume of distribution (Vd) generally has relatively good tissue distribution compared with a drug of restricted distribution. The utility of viewing half-life as a function of the Vd and clearance $(\mathrm{CL})$ is that these two independent parameters reflect the underlying physiology of the animal. Finally, another determinant of the elimination half time for slowly absorbed drugs administered extravascularly is the rate of absorption. If this process is lower than elimination half-life, functionally becomes the biological half time. With depot and sustained release preparations, this phenomenon takes on increased importance to residue avoidance [55].

The problem facing veterinarians is that most pharmacokinetic parameters have been determined in healthy animals. Yet diseased animals would be expected to altered physiology. The half-life will increase if CL is reduced due to an increased Vd. This would result in increased elimination a half-life by a factor of six. The disease may also prolong absorption half-life (decreased blood perfusion of muscles, altered gastrointestinal transit time, etc.) to the point that the elimination profile is different from normal animals. Based on this simplified overview of basic pharmacokinetic principles, the relationship of half-life of the withdrawal time can use to reduce the incidence of violative residues. Doubling dose of the drug should only prolong the approved withdrawal time by one half-life; however, doubling the half-life as a result of the disease would double the necessary withdrawal time pathophysiologic states that increased Vd and/or CL would be expected to prolong half-life [55].

\section{Control and preventive measures}

In the EU, self-monitoring and the control of residues are based on standardised analytical methods. The regulatory framework in force in the EU is based on Directive 96/23/EC, which structures the network of laboratories approved for official residue control, laying down requirements in terms of quality and performance of analytical methods (Decision 2002/657) [56]. In general, the residue control strategy is based on a two-step approach: (1) the detection of residues using sensitive tests with a low rate of false negatives; (2) followed by confirmation, requiring quantification against the MRL and identification with a low rate of false positives [57]. Hence, the residue prevention strategy is based on preventing entry of violative residues in meat or milk intended for human consumption by proper drug use guide developed for use by both veterinarians and food animal (dairy and beef) producers include the following:

Herd health management; all food animals should be maintained in a clean and healthy environment whenever possible. Drug residues are best avoided by implementing management practice (good nutritional to meet growth, maintenance and lactation needs) and herd health program that keep animals healthy and producing efficiently;

Use of approved drugs; dairy and beef producers should not use or store un- approved drugs, special mixes, or products within adequate labels as unapproved drugs have no data regarding efficacy, safety, or withholding time. The herd veterinarian should be certain that ELU involves only approving products;

Establishment of valid veterinarian-client-patient relationship; the use of prescription drug and the ELU necessitate a veterinary-clientpatient relationship, which is established hence a veterinarian is closely with the owner in health management of the herd;

Proper drug administration and identification of treated animals; before administering or dispensing drugs one has to: know the drugs approved for all classes of cattle on the farm and be familiar with approved dosage, route of administration, and withholding time;

Proper maintenance of treatment records and identification of treated animals; institute a workable health record for each animal to record all health related events, including administration of medication. Record the identification of all animals in the permanent health record book;

Having proper drug residue testing capabilities really available on and off the farm; this control point address the conditions under which residue testing should be considered; the proper selection and interpretation of tests; the inherent limitation and potential misuse of residue testing; and

Creating awareness of proper drug use, and methods to avoid marketing adulterated products principally educational, total residue avoidance program is based upon the objective of improving the livestock producer's management and quality control of marketing animals with emphasis on avoidance of drug residues [58-60].

\section{Conclusion and Recommendations}

The use of veterinary drugs in food-producing animals has the potential to generate residues in animal derived products and poses a health hazard to the consumer. Veterinarians are facing a dramatic change in attitude and behaviours concerning drug residues because of the therapeutic and prophylactic use of drugs. Until recently, veterinarians did not pay sufficient attention to ensuring that the producers adhered strictly to the withdrawal period for milk, meat, and egg from animals treated with a variety of drugs. The most likely reason for drug residues may result from human management, such as improper usage, including extra-label or illegal drug applications. However, the most obvious reason for unacceptable residues might be due to failure to keep to the withdrawal period, including using overdose and long-acting drugs. There is also limited information on the magnitude of veterinary drug residue worldwide. Hence, an extensive work has to be carried out to prevent the occurrence of VMPs residues and to familiarize all animal health professionals with 
the knowledge of pharmacokinetics, pharmacodynamics and toxicological effects of pharmaceutical preparations that are useful in prevention, control, and treatment of animal disease as specified times required for withdrawal of medication from food of animal origin prior to ready for human consumption.

\section{Competing interests}

The author declares that they have no competing interests.

\section{References}

1. Environment and human health (EPA) (2015) Environmental Protection Agency.

2. Ames BN (1983) Dietary carcinogens and anticarcinogens. Oxygen radicals and degenerative diseases. Science 221: 1256-1264.

3. Rokka M, Eerola S, Perttilä U, Rossow L, Venäläinen E, et al. (2005) The residue levels of narasin in eggs of laying hens fed with unmedicated and medicated feed. Mol Nutr Food Res 49: 38-42.

4. Crawford LM (1985) The impact of residues on animal food products and Human Health. Rev Sci tech Off int Epiz 4: 669-685.

5. Antimicrobial use and antimicrobial resistance (2015) American Veterinary Medicine Association (AVMA).

6. Health Canada (2013) Maximum residue limits for veterinary drugs.

7. Takele B, Abdulkaf K, Tariku J, Fanos T, Dinka A, et al. (2015). Assessement on chemicals and drugs residue in dairy and poultry products in Bishoftu and Modjo, central Ethiopia. J Nutri Food Sci S13: 003.

8. Commission staff working document on the implementation of national residue monitoring plans in the member states in 2009 (2010) European Commission (EC) Council Directive.

9. Boothe DM, Reevers PT (2012) Pharmacology introduction In: Aiello SE Moses MA, Steigerwald MA (Eds) The Merck Veterinary Manual. Merck Publishing Group.

10. International Cooperation on Harmonization of technical Requirements for registration of veterinary medicinal products (VICH) (2011) Studies to evaluate the metabolism and residue kinetics of veterinary drugs in food-producing animals: metabolism study to determine the quantity and identify the nature of residues. VICH GL 46 (MRK)-Metabolism and Residue Kinetics.

11. Rico AG, Burgat-Sacaze V (1985) Veterinary drugs and food safety: a toxicological approach. Rev Sci tech int Epiz 4: 111-119.

12. Doyle ME (2006) Veterinary drug residues in processed meats-potential health risk. FRI briefings, Food Research Institute, University of Wisconsin-Madison, Madison.

13. Canadian Food Inspection Agency (CFIA) (2014) Chemical residues. In: Meat Hygiene Manual of procedures: Chapter 5-Sampling and Testing Procedures. Meat Hygiene Directives.

14. Beyene T, Tesega B (2014) Rational veterinary drug use: Its significance in public health. J Vet Med Anim Health 6: 302-308.

15. Schwark W S (2014) Factors that affect drug disposition in foodproducing animals during maturation. Department of Pharmacology. New York State College of Veterinary Medicine, Cornel University, 70:3635-3645

16. Gips M, Soback S (1996) Norfloxacin nicotinate pharmacokinetics in unweaned and weaned calves. J Vet Pharmacol Ther 19: 130-134.

17. Kaneene JB, Miller R (1997) Problems associated with drug residues in beef from feeds and therapy. Rev Sci Tech 16: 694-708.

18. Bushra R, Aslam N, Khan AY (2011) Food-drug interactions. Oman Med J 26: 77-83.

19. Toutain PL, Ferran A, Bousquet-Me'lou A (2010) Species Differences in Pharmacokinetics and Pharmacodynamics. In: Cunningham F, Elliot J, Lees P (Eds) Comparative and Veterinary Pharmacology: Handbook of Experimental Pharmacology, Springer-Verlag Berlin Heidelberg,pp. 199.
20. McConville ML, Dijkstra JW, Stamm JM, van Saene JJ, Nouws JF (1995) Effects of sarafloxacin hydrochloride on human enteric bacteria under simulated human gut conditions. Vet Q 17: 1-5.

21. Korsrud GO, Boison JO, Papich MG, Yates WD, MacNeil JD, et al. (1993) Depletion of intramuscularly and subcutaneously injected procaine penicillin $\mathrm{G}$ from tissues and plasma of yearling beef steers. Can J Vet Res 57: $223-230$

22. Riviere JE, Craigmill AL, Sundlof SF (1991) Handbook of comparative pharmacokinetics and residues of veterinary antimicrobials. Boca Raton, FL (Ed) CRC press, Inc, Florida, USA.

23. Botsoglou NA, Fletouris DJ (2001) Drug residues in foods pharmacology: food safety, and analysis. Marcel Dekker (Ed) New York, USA pp. 98-150.

24. Weaver L (1992) Antibiotic residues in milk and meat: perceptions and realities. Vet Med 12: 1222-1228.

25. Gillian C (2003) Avoiding drug carry over during feed processing and delivery. FDA Veterinarian Newsletter 6: 30-45.

26. Code of Federal Regulations (CFR) (2006) Food and Drug Administration.

27. Jacela JY, DeRouchey JM, Tokach MD (2009) Feed additives for swine Fact sheets-acidifiers and antibiotics. J Swine Health Prod 17: 270-275.

28. Zuo XN, Xu T, Jiang L, Yang Z, Cao XY, et al. (2012) Toward reliable characterization of functional homogeneity in the human brain preprocessing, scan duration, imaging resolution and computational space. Epub 65: 374-386.

29. Babapour A, Azami L, Fartashmehr J (2012) Overview of Antibiotic Residues in Beef and Mutton in Ardebil, North West of Iran. World Applied Sciences Journal 19: 1417-1422.

30. Ozbay G, Babu BK, Chen G (2013) Prevalence of veterinary drug residues and heavy metals in catfish nuggets. Journal of Food Processing \& Technology S11: 1-7.

31. Kehinde OG, Junaidu K, Mohammed M, AbdulRahman AM (2012) Detection of Antimicrobial Drug Residues in Commercial Eggs Using Premi Test. International Journal of Poultry Science 11: 50-54.

32. Ibrahim A, Junaidu A, Garba M (2009) Multiple antibiotic residues in meat from slaughtered cattle in Nigeria. The Internet Journal of Veterinary Medicine 8: 1.

33. Desalegne A, Kelay B, Girma Z (2014) Detection and determination of Oxytetracycline and Penicillin G antibiotic residue levels in bovine bulk milk from Nazareth dairy farms, Ethiopia. Ethiop Vet J 18: 1-15.

34. Addisalem HB, Bayleyegn MZ, Bayleyegn MZ (2012) Tetracycline Residue Levels in Slaughtered Beef Cattle from Three Slaughterhouses in Central Ethiopia. Global Veterinaria 8: 546-554

35. Darwish WS, Eldaly EA, El-Abbasy MT, Ikenaka Y, Nakayama S, et al. (2013) Antibiotic residues in food: the African scenario. Jpn J Vet Res 61 Suppl: S13-22.

36. Joint FAO/WHO Expert Committee on Food Additives (JECFA) (2013) Residue evaluation of certain veterinary drugs. 8th Meeting, FAO/ JECFA Monographs, pp. 15.

37. Samanidou V, Nisyriou S (2008) Multi-residue methods for confirmatory determination of antibiotics in milk. J Sep Sci 31: 2068-2090.

38. Food Safety Authority of Ireland (FSAI) (2007) Prudent use of medicines, pesticides and chemicals on farms.

39. Chang Q, Wang W, Regev-Yochay G, Lipsitch M, Hanage WP (2014) Antibiotics in agriculture and the risk to human health: how worried should we be? Evolutionary Applications, 8: 240-247.

40. Landers TF, Cohen B, Wittum TE, Larson EL (2012) A review of antibiotic use in food animals: perspective, policy, and potential. Public Health Rep 127: 4-22.

41. National Research Council (NRC) (1991) The use of drugs in food animal benefits and risks. The National academies Press 253: 284

42. Riedl MA, Casillas AM (2003) Adverse drug reactions: types and treatment options. Am Fam Physician 68: 1781-1790.

43. American Cancer Society (ACS) (2014) Known and probable human carcinogens. 
Citation: Beyene T (2016) Veterinary Drug Residues in Food-animal Products: Its Risk Factors and Potential Effects on Public Health . J Veterinar Sci Technol 7: 285. doi:10.4172/2157-7579.1000285

Page 7 of 7

44. Aiello SE, Lines PR, Kehn CM (2005) Anthelmintics In: The Merck Veterinary Manual. (9th Edn) Co.Ioc.NJ, USA, pp: 2111-2124.

45. Brown TA (2002) Mutation, Repair and Recombination. In: Genomes. (2nd Edn) Garland Science, Manchester, UK.

46. Booth NH, Mc Donald LE (1988) Toxicology of drug and chemical residues. In: veterinary pharmacology and therapeutics. (6thedn) Iowa state university press, USA pp: 1149-1195.

47. Foster W, Beecroft ML (2014) Chemical Exposures and Human Fertility. Infertility Awarness Association of Canada.

48. El-Makawy A, Radwan HA, Ghaly IS, El-Raouf AA (2006) Genotoxical, teratological and biochemical effects of anthelmintic drug oxfendazole Maximum Residue Limit (MRL) in male and female mice. Reprod Nutr Dev 46: 139-156.

49. Cotter PD, Stanton C, Ross RP, Hill C (2012) The impact of antibiotics on the gut microbiota as revealed by high throughput DNA sequencing. Discov Med 13: 193-199.

50. Jackson BA (1980) US Food and Drug Administration, Center for Veterinary Medicine. Hosted at University of Nebraska Lincoln. Jam Vet Med Assoc pp: 176: 1141.

51. European Community (EC) (2001) Notice to applicant and note for guidance. Establishment of maximum residue limits for residue of veterinary medicinal products in food stuffs of animal origin pp. 4-10.

52. Boisseau J (1993) Basis for the evaluation of the microbiological risks due to veterinary drug residues in food. Vet Microbiol 35: 187-192.

53. Food and Drug administration Center for Veterinary Medicine (FDACVM) (2006) Guidance for approval of a withdrawal period. In
Contains-Binding Recommendations: Guidance for Industry- General Principles for evaluating the safety of compounds used in food-producing animals. U.S. Department of Health and Human Services.

54. Apley M (2003) How do violative residues happen in swine? Pork Safety Sheet. National Pork Board, Iowa State University. USA.

55. International Dairy Federation (IDF) (1995) Symposium on residues of antimicrobial drugs and other inhibitors in milk. In: Proceedings of a joint conference of IDF, AOAC. International and the German National Committee of IDF, Germany.

56. European Commission (EC) (2002) Commission Decision of 12 August 2002 implementing Council Directive 96/23/EC concerning the performance of analytical methods and the interpretation of results. Off $\mathrm{J}$ Eur Communities 221: 8-36.

57. Mensah SE, Koudandé OD, Sanders P, Laurentie M, Mensah GA, et al. (2014) Antimicrobial residues in foods of animal origin in Africa: public health risks. Rev Sci Tech 33: 987-996, 975-86.

58. American Veterinary Medicine Association (AVMA) and National Milk Production Fed-eration (NMPF) (1991) Milk and beef residue prevention: a quality assurance protocol. J Ame Vet Assoc 2: 1-24.

59. Gerald BG, Joseph CP (1991) Report from symposium on prevention of unwanted Drug Residues. J Ame Vet Med Assoc 26: 89-111.

60. Scippo ML, Degand G, Duyckaerts A, Maghuin-Rogister G, Delahaut P (1994) Control of the illegal administration of natural steroid hormones in the plasma of bulls and heifers. Analyst 119: 2639-2644. 